\title{
NLRP6 as a Negative Regulator of Innate Immunity
}

\author{
Zahid Manzoor and Young-Sang Koh ${ }^{*}$ \\ Department of Microbiology and Immunology, Jeju National University School of Medicine, Jeju, Korea
}

\begin{abstract}
Nucleotide-binding and oligomerization domain (NOD)-like receptors (NLRs) play important role in immune responses by regulating nuclear factor kappa-light-chain-enhancer of activated B cells (NF-kB), type I interferon and inflammasomes. Nod-like receptor pyrin domain-containing protein 6 (NLRP6) is vital for protection of mice from colitis and colorectal tumorigenesis. However, the role of NLRP6 in microbial infection remains unclear. NLRP6 was recently reported as negative regulator of inflammatory signaling and demonstrated that how it hinders clearance of bacterial pathogens.
\end{abstract}

Key Words: Nod-like receptor pyrin domain-containing protein 6, Inflammasome, Listeria monocytogenes

In Nature on $16^{\text {th }}$ August 2012, Anand et al reported that Nod-like receptor pyrin domain-containing protein 6 (NLRP6) negatively regulates innate immunity and host defense against bacterial pathogens (1). Pattern recognition receptors, such as Toll-like receptors (TLRs) or NLRs recognizes highly conserved molecular patterns exclusively present in microbes. TLRs detect pathogens in extracellular or in endosomes compartment, while NLRs are involved in intracellular surveillance $(2,3)$. Inflammasome is a cytoplasmic multiprotein structure composed of one of several NLR proteins that can be activated by variety of stimuli including bacterial toxins, Adenosine triphosphate or crystals (4). Inflammasomes play role in activation of caspase-1, which proteolytically process and release the cytokines interlukin (IL)-1 $\beta$ and IL-18, and instigate pyroptosis; a lytic host cell death. Some NLRs including NLRP1, NLRP3 and NLRC4 facilitate the activation of inflammatory caspases in inflammasomes while others such as NOD1 and NOD2 contribute to host defense against pathogen by activation of nuclear factor kappa-light-chain- enhancer of activated B cells (NF- $\mathrm{BB}$ ) and mitogen-activated protein kinases (MAPKs) signaling (4). NLRP6 is a member of the NOD-like receptor (NLR) family of intracellular proteins that plays vital role in innate immunity $(1,5)$. Deficiency of NLRP6 in mice results in spontaneous intestinal hyperplasia and inflammatory cell recruitment. In addition NLRP6 deficient mice resulted in reduced levels of IL-18 and altered fecal microbiota (6). NLRP6 contributes to protection against colitis, inflammatory bowel disease and colorectal tumorigensis by altering the composition of gut microflora $(5,6)$. Nlrp $6^{--}$mice has drastic consequences on the composition of the microbial communities that leads to spontaneous and induced colitis (6).

Anand et al showed that mice lacking NLRP6 are highly resistant to infection with bacterial pathogens Listeria monocytogens, Salmonella typhimurium and Escherichia coli (1). Nlrp $6^{-1-}$ mice showed more rapid clearance of bacterial infection as compared to wild type mice, suggesting that NLRP6 hinders bacterial clearance (1). Here we summarize their results that NLRP6 negatively

Received: January 21, 2013/ Revised: January 23, 2013/ Accepted: January 28, 2013

${ }^{*}$ Corresponding author: Young-Sang Koh. Department of Microbiology and Immunology, Jeju National University School of Medicine, 102 Jejudaehakno, Jeju 690-756, Korea.

Phone: +82-64-754-3851, Fax: +82-64-702-2687, e-mail: yskoh7@jejunu.ac.kr

** This research was supported by the 2013 scientific promotion program funded by Jeju National University.

(c) This is an open access article distributed under the terms of the Creative Commons Attribution Non-Commercial License (http://creativecommons.org/license/by-nc/3.0/). 
regulate inflammatory signaling and hinders the clearance of both Gram-positive and -negative bacteria (1).

Anand et al showed that all the wild-type mice surrender to infection within $5 \sim 6$ days while $75 \%$ of $N l r p 6^{--}$mice survived the infection (1). As compared to wild-type, Nlrp $6^{-\alpha}$ mice represented significantly less number of bacterial dissemination at day 1 and 3 after infection (1). In agreement, immunohistochemical analysis of liver sections revealed that $L$. monocytogenes were absent from liver capsules of $N$ lrp $6^{-1}$ mice as compared to wild-type (1). Recently it has been shown that Nlrp $6^{-}$mice have different microflora composition which is transferable to co-housed wild-type mice (6). Co-housing wild-type and $N \operatorname{lrp} 6^{-1}$ mice for 4 weeks resulted in equalized microflora prevalence in these mice (1). However, co-housing of these mice did not showed any effect on resistant phenotype of Nlrp $6^{-}$mice to L. monocytogenes infection as determined by bacterial load in spleen and liver. They concluded that NLRP6 regulates L. monocytogenes independent of its microbiota (1). Nlrp $6^{-}$mice were not only resistant to $L$. monocytogenes rather they also showed resistance against S. typhimurium and E. coli as determined by significantly lower bacterial burden in liver and spleen (1). These results suggested that NLRP6 may not only promote systemic dissemination and colonization of both Gram-positive and -negative bacteria rather intracellular and extracellular bacteria as well in hosts (1).

Infection results in movement of innate immune cells, mainly monocytes and neutrophils from the bone marrow to the circulation to be deployed at the site of infection (2). The phagocytosed infectious agents by immune cells are degraded by fusion with acidic lysosomes (2). By using automated haematology analyzer monocytes and neutrophils in peripheral blood of wild-type and Nlrp $6^{-}$mice were determined. They found significantly higher number of circulating monocytes and neutrophils in L. monocytogenes infected Nlrp $6^{-1}$ mice as compared to wild-type (1). For further confirmation of these results they analyzed the infiltration of immune cells in the peritoneal cavity of $L$. monocytogenes infected Nlrp $6^{-1}$ and wild-type mice (1). Infiltration of monocytes and neutrophils in peritoneal cavity was significantly higher in L. monocytogenes infected $N l r p 6^{--}$mice as compared to wild-type (1). These results suggest that NLRP6 regulates the influx of monocytes and neutrophils to the circulation and to peritoneum during bacterial infections (1).

Upon infection, innate immune cells produce immediate immune response by activating NF- $\mathrm{\kappa B}$ and MAPK pathways which ultimately provide robust immunity (2). NLRP6 regulates the NF- $\mathrm{KB}$ and MAPK signaling pathways as determined by measuring the phosphorylation of extracellular signal-regulated kinases 1 and 2 (ERK1/2) and IKB (1). Infected $N l r p 6^{-}$macrophages showed significantly increased levels of phospho-ERK1/2, and failed to downregulate phospho-IKB, suggesting that NLRP6 may negatively regulate NF- $\mathrm{KB}$ and ERK activation downstream of TLRs. For further confirmation of these results they measured the $\mathrm{NF}-\mathrm{KB}$ and MAPK dependant production of cytokines and chemokines (1). Nlrp $6^{-}$macrophages infected with $L$. monocytogenes showed significantly higher secretion of tumor necrosis factor, IL-6 and keratinocyte chemoattractant (1). The serum and peritoneal fluid levels of IL-6 and keratinocyte chemoattractant were higher in infected Nlrp $6^{-1}$ mice as compared to wild-type. Thus they concluded that NLRP6 regulates TLR-induced MAPK and NF- $\mathrm{kB}$ signaling to diminish the production of pro-inflammatory cytokines and chemokines during bacterial infections (1).

Anand et al showed that NLRP6 negatively regulates inflammatory signaling and demonstrated that it hinders the clearance of both Gram-positive and -negative bacterial pathogens. Although they concluded form their study that NLRP6 negatively regulates TLR-induced MAPK and NF-KB signaling, they suggested further detailed study to determine the precise level at which NLRP6 negatively regulates TLR-induced responses. They also proposed that NLRP6 may be essential for diminishing the production of pro-inflammatory cytokines under conditions in which strong inflammatory responses might be harmful to host.

\section{REFERENCES}

1) Anand PK, Malireddi RK, Lukens JR, Vogel P, Bertin J, 
Lamkanfi M, et al. NLRP6 negatively regulates innate immunity and host defence against bacterial pathogens. Nature 2012;488:389-93.

2) Anand PK, Kanneganti TD. Targeting NLRP6 to enhance immunity against bacterial infections. Future Microbiol 2012;7:1239-42.

3) Hong S, Park S, Yu JW. Pyrin domain (PYD)containing inflammasome in innate immunity. J Bacteriol Virol 2011;41:133-46.

4) Elinav E, Strowig T, Henao-Mejia J, Flavell RA.
Regulation of the Antimicrobial Response by NLR Proteins. Immunity 2011;34:665-79.

5) Chen GY, Liu M, Wang F, Bertin J, Núñez G. A functional role for Nlrp6 in intestinal inflammation and tumorigenesis. J Immunol 2011;186:7187-94.

6) Elinav E, Strowig T, Kau AL, Henao-Mejia J, Thaiss CA, Booth CJ, et al. NLRP6 inflammasome regulates colonic microbial ecology and risk for colitis. Cell 2011; 145:745-57. 